\title{
Prevention and Treatment of CMV Retinitis Related Blindness in Resource-Limited Settings, Myanmar
}

\author{
Ni Ni Tun ${ }^{1^{*}}$, Thida Yamin Pyone ${ }^{1}$, Yee Yee Aung ${ }^{1}$ and Frank Smithuis ${ }^{1,2}$ \\ ${ }^{1}$ Medical Action Myanmar, Yangon, Myanmar \\ ${ }^{2}$ Myanmar Oxford Clinical Research Unit, Yangon, Myanmar
}

*Corresponding author: Ni Ni Tun, E-mail: nini@mam.org.mm

\section{Introduction}

In south-east Asia, cytomegalovirus (CMV) retinitis is a neglected disease [1], with no defined strategy for management. This is despite evidence that $\mathrm{CMV}$ retinitis is a common cause of HIV-associated blindness in this region and the second most common opportunistic infection to emerge during initiation of antiretroviral therapy (ART) and that CMV viremia is a strong predictor of mortality. The emerging body of data from resource-limited settings closely mirrors what was learned several decades ago in western countries about CMV infection in patients with AIDS before ART became available. At that time, about one-third of patients with AIDS developed CMV retinitis, accounting for more than $90 \%$ of cases of HIV-related blindness. Furthermore, extra-ocular CMV disease was a major cause of AIDS-related morbidity and mortality. In resource-limited settings, the management of $\mathrm{CMV}$ is hardly available. Primary care clinicians have been reluctant to engage in the care of CMV retinitis, partly because of inadequate training in diagnostic approaches, partly because oral valganciclovir, the treatment of choice, is expensive and therefore usually not available, and partly because the alternative treatment intraocular injection with ganciclovir-viewed as a procedure that only an ophthalmologist could perform safely. But ophthalmologists are limited in number and often distant from the need, based in urban secondary or tertiary care facilities. In addition, many ophthalmologists in developing countries may lack the skills or equipment for adequate management of $\mathrm{CMV}$ retinitis. The end result is that when patients are referred to ophthalmologists they commonly arrive when the disease is at a late stage and outcomes are poor. Thus, there is a need for a simple and effective management of $\mathrm{CMV}$ retinitis that can be implemented at the primary care level.

Myanmar is one of the poor countries in south east Asia where HIV prevalent is highly concentrated in key affected populations such as intravenous drug users $(28.5 \%)$, sex workers $(14.6 \%)$ and men who have sex with men (11.6\%) [2] and limited ART availability. Training of HIV clinicians to do early diagnosis of CMV retinitis by using indirect ophthalmoscopy [3] and intraocular ganciclovir injection was initiated on a small scale since 2006 [4] and regular training and monitoring happened ever since by Médecins Sans Frontières and Medical Action Myanmar.

\section{Case Report}

A young woman aged 24 years was diagnosed as HIV positive on $12^{\text {th }}$ March 2016 and her CD4 count was 15 cells/microliter. Screening for opportunistic infections including screening for cytomegalovirus retinitis by indirect ophthalmoscope was done on $25^{\text {th }}$ March 2016, and no abnormal finding was detected in both eyes which had normal eye vision of $6 / 6$ on both sides. She was diagnosed as pulmonary tuberculosis and anti TB treatment was provided.

On $24^{\text {th }}$ April ART was started with Tenofovir, lamivudine and efaverance fixed dose combination. Follow up screening of CMV eye infection was done on $29^{\text {th }}$ April and no abnormal finding was found in both eyes with normal eye vision (6/6) on both sides. Two weeks after commenc-

Citation: Tun NN, Pyone TY, Aung YY, Smithuis F (2018) Prevention and Treatment of CMV Retinitis Related Blindness in Resource-Limited Settings, Myanmar. Clin Med Rev Case Rep 5:238. doi. org/10.23937/2378-3656/1410238

Accepted: October 19, 2018: Published: October 21, 2018

Copyright: (C) 2018 Tun NN, et al. This is an open-access article distributed under the terms of the Creative Commons Attribution License, which permits unrestricted use, distribution, and reproduction in any medium, provided the original author and source are credited. 


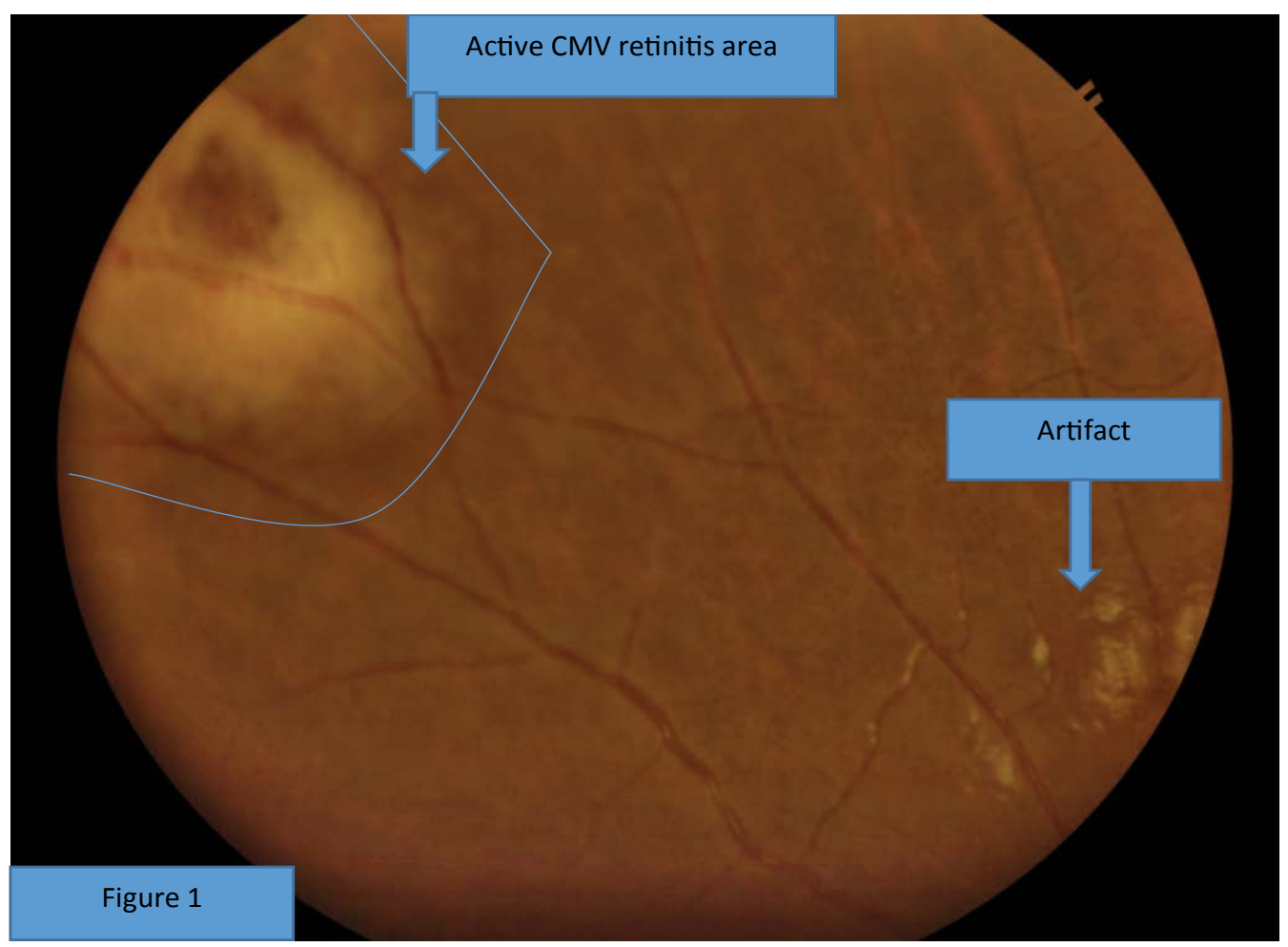

Figure 1: Active CMV retinitis: Characterized by dense retinal whitening lesion with irregular border of multiple small white satellite lesions.

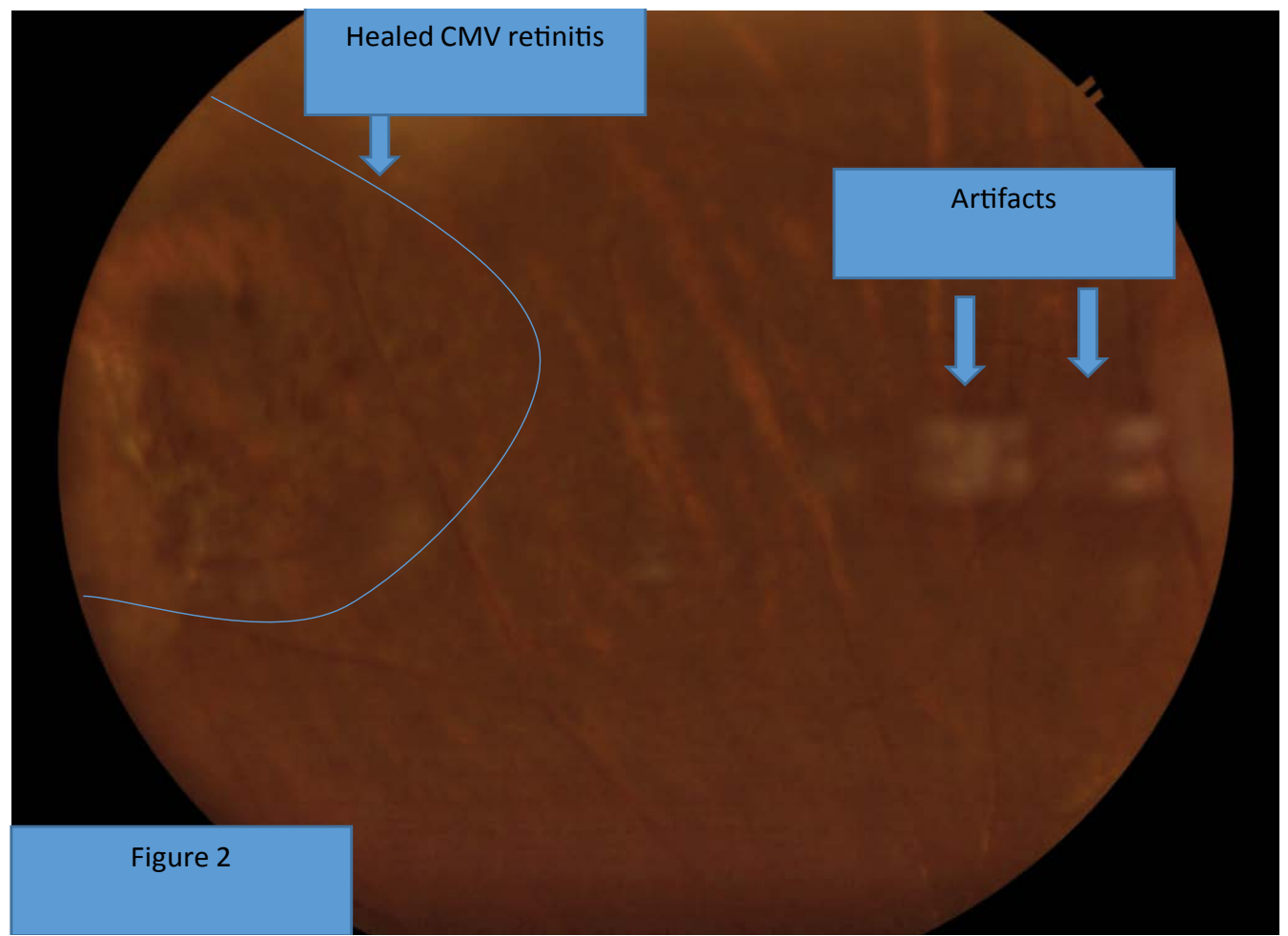

Figure 2: Healed CMV retinitis: Characterized by pigmented scar, healed with well-defined margin (no satellite lesion at the border).

ing ART (12 May), a small dense white retina lesion was found in her left eye and a differential diagnosis of cotton wool spot (which is common in advanced immunocompromised patients) and early CMV lesion was made. So, subsequent follow up of eye screening was done on 24 May and active $\mathrm{CMV}$ retinitis was diagnosed (Figure 1- Characterized by dense retinal whitening, irregular border and small white satellite lesions [1]). Eye vision was $6 / 6$ on both eyes.

Intraocular gancyclovir injection to left eye was given (valganciclovir was unavailable at that time) and ART was continued. After 3 weekly intraocular ganciclovir injections the lesion became inactive and the injections were stopped (Figure 2). The patient was observed for 
CMV infection by indirect ophthalmoscope every two weeks until her CD4 rose up to more than 100 cells/ microliter. After that, $\mathrm{CMV}$ retinitis screening was done every 6 months up to present. Serological diagnosis of CMV infection was not available in the country. Now the patient is more than two years on ART. Her last CD4 count was 332 cells/microliter and the lesion is in inactive stage.

\section{Discussion}

Cytomegalovirus (CMV) is a member of the herpes virus family. It is usually acquired in childhood and over $90 \%$ of adults living in resource-limited settings in middle and low income countries are positive. CMV remains latent for life, but can become active if the immune system becomes significantly impaired. In the setting of HIV infection, cytomegalovirus (CMV) can affect both the central and peripheral nervous systems. Neurological manifestations of CMV infection include encephalitis, ventriculitis, myelitis, retinitis, radiculoganglionitis, and peripheral neuropathies. CMV infects the brain or spinal cord, meninges, or nerve roots. It can also cause colitis, esophagitis, adrenalitis, hepatitis and pneumonitis. This usually occurs in patients with severe immunodeficiency: $\mathrm{CD} 4+$ lymphocyte counts typically are less than $50 / \mathrm{mm}^{3}$.

CD4 counts are known to have biological variation, and automated CD4 counts have variations of $10-20 \%$, hence, the screening cut off has been set at $<100 / \mathrm{mm}^{3}$. $\mathrm{CMV}$ retinitis caused over $90 \%$ of AIDS-related blindness in developed countries before ART therapy was introduced, and now causes over $90 \%$ of AIDS-related blindness in Myanmar, as well as the rest of South East Asia, China, India and Eastern Europe (Russia and the Ukraine). Untreated, CMV retinitis is relentlessly progressive and will destroy the entire retina in 4-6 months. However, blindness commonly occurs earlier, from complications such as destruction of the optic nerve or macula, retinal detachment, and Immune Recovery Uveitis (IRU). In resource-limited settings, the patient generally does not complain of symptoms until vision loss has occurred, when the damage is already permanent and not reversible. Clinical symptoms that a patient may complain of are blurred vision, floaters, scotoma (holes in the vision or blind areas) or flashing lights in the eye. The "gold standard" for diagnosis is indirect ophthalmoscopy through a fully dilated pupil by a trained clinician. Successful treatment of CMV in patients with AIDS requires both, ART and specific medication against CMV. Valganciclovir, an oral version of ganciclovir is the ideal systemic CMV treatment, covering both ocular and extra-ocular disease, but is very costly and at the moment unavailable to us. At this time the most cost effective treatment is intraocular ganciclovir injections. This treatment in combination with ART will effectively stop the progression of $\mathrm{CMV}$, and therefore also limit the occurrence of secondary blinding complications (retinal detachment and IRU) which both increase in frequency in direct proportion to the area of retina that is infected.

The vision loss from CMV is commonly profound or total, and permanent, which means that the patient will require near constant assistance from another individual and retain little or no capacity for independence. Early diagnosis and treatment is therefore of great importance.

To enable early diagnosis, all patients with CD4 $<100$ cells $/ \mathrm{mm}^{3}$ should be referred for indirect ophthalmoscopy without delay and should be screened within the first week of presentation for care.

\section{References}

1. Heiden D, Ford N, Wilson D, Rodriguez WR, Margolis T, et al. (2007) Cytomegalovirus retinitis: The neglected disease of the AIDS pandemic. PLoS Med 4: 1845-1851.

2. http://aidsdatahub.org/national-strategic-plan-hiv-and-aidsmyanmar-2016-2020-ministry-health-and-sports-myanmar-2017

3. Heiden D, Tun N, Maningding E, Heiden M, Rose-Nussbaumer J, et al. (2014) Training clinicians treating HIV to diagnose cytomegalovirus retinitis. Bull World Health Organ 92: 903-908.

4. Tun N, London N, Kyaw M, Smithuis F, Ford N, et al. (2011) CMV retinitis screening and treatment in a resource-poor setting: Three-year experience from a primary care HIV/ AIDS programme in Myanmar. J Int AIDS Soc 14: 41. 